\title{
Radial segments and conformal mapping of an annulus onto domains bounded by a circle and a $k$-circle
}

\author{
by Tetsuo Inoue (Kobe)
}

Tadao Kubo in memoriam

\begin{abstract}
Let $f(z)$ be a conformal mapping of an annulus $A(R)=\{1<|z|<R\}$ and let $f(A(R))$ be a ring domain bounded by a circle and a $k$-circle. If $R(\varphi)=\{w: \arg w=\varphi\}$, and $\ell(\varphi)-1$ is the linear measure of $f(A(R)) \cap R(\varphi)$, then we determine the sharp lower bound of $\ell\left(\varphi_{1}\right)+\ell\left(\varphi_{2}\right)$ for fixed $\varphi_{1}$ and $\varphi_{2}\left(0 \leq \varphi_{1} \leq \varphi_{2} \leq 2 \pi\right)$.
\end{abstract}

1. Introduction. We denote the chordal distance between the points $w_{1}$ and $w_{2}$ in the extended complex $w$-plane $\overline{\mathbb{C}}$ by $q\left(w_{1}, w_{2}\right)$, that is,

$$
q\left(w_{1}, w_{2}\right)=\left|w_{1}-w_{2}\right| / \sqrt{\left(1+\left|w_{1}\right|^{2}\right)\left(1+\left|w_{2}\right|^{2}\right)}
$$

if $w_{1}$ and $w_{2}$ are both finite, and

$$
q\left(w_{1}, \infty\right)=1 / \sqrt{1+\left|w_{1}\right|^{2}}
$$

We define the chordal cross ratio of quadruples $w_{1}, w_{2}, w_{3}, w_{4}$ in $\overline{\mathbb{C}}$ by

$$
X\left(w_{1}, w_{2}, w_{3}, w_{4}\right)=\frac{q\left(w_{1}, w_{2}\right) q\left(w_{3}, w_{4}\right)}{q\left(w_{1}, w_{3}\right) q\left(w_{2}, w_{4}\right)} .
$$

A Jordan curve $\Gamma$ in $\overline{\mathbb{C}}$ is called a $k$-circle, where $0<k \leq 1$, if for all ordered quadruples of points on $\Gamma$,

$$
X\left(w_{1}, w_{2}, w_{3}, w_{4}\right)+X\left(w_{2}, w_{3}, w_{4}, w_{1}\right) \leq 1 / k .
$$

This definition of a $k$-circle was introduced by Blevins [2]. It is well known that a $k$-circle is a quasicircle (see [1]). One of the simplest $k$-circles is $\{w:|\arg w|=\arcsin k\}$. Throughout the note the value of $\arcsin$ and arccos is restricted between 0 and $\pi / 2$.

In this note we consider the class $C(k)$ of conformal mappings $w=$ $f(z)$ of an annulus $A(R)=\{1<|w|<R\}$ whose images $D_{f}=f(A(R))$

1991 Mathematics Subject Classification: Primary 30C25. 
are ring domains with inner boundary $f(|z|=1)=\{|w|=1\}$ and outer boundary $\Gamma$ a $k$-circle. Let $R(\theta)=\{w: \arg w=\theta\}$ and let $\ell(\theta)-1$ be the linear measure of $R(\theta) \cap f(A(R))$. Let $D\left(k, d_{0}\right)$ be the ring domain with $\operatorname{Mod} D\left(k, d_{0}\right)=\log R$ and with inner boundary $\{|w|=1\}$ and outer boundary $\left\{w:\left|\arg \left(w+d_{0}\right)\right|=\pi-\arcsin k\right\}$. Let $f_{0}(z)$ be a function mapping $A(R)$ onto $e^{i \beta} D\left(k, d_{0}\right)$ and set

$$
T(w)=\frac{w_{1}}{\bar{w}_{1}} \cdot \frac{1+\bar{w}_{1} w}{w+w_{1}}
$$

where

$$
\begin{gathered}
\beta=\arcsin \left(\sin \theta /\left(d_{0}\left(d_{1}+\sqrt{d_{1}^{2}-1}\right)\right)\right), \\
w_{1}=\left(d_{1}+\sqrt{d_{1}^{2}-1}\right) e^{i \theta}, \quad d_{1}=\sqrt{d_{0}^{2} \cos ^{2} \theta+\sin ^{2} \theta} .
\end{gathered}
$$

We show the following theorem dealing with radial segments.

THEOREM. Under the above assumptions, we have the inequalities

$$
\ell(\theta)+\ell(\pi-\theta) \geq 2\left(d_{1}+\sqrt{d_{1}^{2}-1}\right)
$$

for $0 \leq \theta \leq \arccos \left(\sqrt{d_{0}^{2}-1} /\left(2 d_{0}\right)\right)$, while

$$
\ell(\theta)+\ell(\pi-\theta) \geq 2 d_{0}
$$

for $\arccos \left(\sqrt{d_{0}^{2}-1} /\left(2 d_{0}\right)\right)<\theta \leq \pi / 2$.

For $0 \leq \theta \leq \theta_{0}$, equality is attained only for the function $F(z)=T\left(f_{0}(z)\right)$ up to a rotation around the origin, where $\theta_{0}$ is a positive constant depending only on $k$, and determined in the proof of the theorem.

We remark that this theorem can be reformulated as an estimate for $\ell\left(\varphi_{1}\right)+\ell\left(\varphi_{2}\right)\left(0 \leq \varphi_{1} \leq \varphi_{2} \leq 2 \pi\right)$. For example, (1.3) is equivalent to

$$
\ell\left(\varphi_{1}\right)+\ell\left(\varphi_{2}\right) \geq 2\left(d_{2}+\sqrt{d_{2}^{2}-1}\right)
$$

with $d_{2}=\sqrt{\left(1+d_{0}^{2}+\left(1-d_{0}^{2}\right) \cos \left(\varphi_{2}-\varphi_{1}\right)\right) / 2}$. Let $w=f(z)$ be a conformal mapping of an annulus $A(R)$ (with $\Gamma$ not necessarily a $k$-circle). Mityuk [8] obtained the lower bound of $\ell(\theta)+\ell(\pi+\theta)(0 \leq \theta \leq \pi)$. Our theorem yields his result by considering the special case of $\varphi_{2}-\varphi_{1}=\pi$ and letting $k \rightarrow 0$.

2. Fundamental lemma. In this section we will verify the following fundamental lemma on the Koebe region for the class $C(k)$.

Fundamental Lemma. Let $w=f(z)$ be a function in $C(k)$. Then the distance $d(\Gamma, 0)$ between the origin and $\Gamma$ satisfies the inequality

$$
d(\Gamma, 0) \geq d_{0} .
$$

Equality holds in (2.1) if and only if $D_{f}$ is $D\left(k, d_{0}\right)$ up to a rotation around the origin. 
This lemma can be restated as follows: The Koebe region for the class $C(k)$ is generated by functions $f$ arising from $f_{0}$ by rotations around the origin.

Proof of the fundamental lemma. First we verify this lemma under the condition that $\Gamma=f(|z|=R)$ contains the point at infinity.

Let $w^{\prime}$ be a point on $\Gamma$ such that $\left|w^{\prime}\right|=d(\Gamma, 0)(=a)$. We consider the circular symmetrization $D_{f}^{*}$ of $D_{f}$ with respect to the positive real axis.

The following statement is due to Blevins [2]: If $\Gamma$ contains the point at infinity and a point $w^{\prime}$ with $\left|w^{\prime}\right|=a$, then the circular symmetrization $D_{f}^{*}$ of $D_{f}$ with respect to the positive real axis is contained in the domain $D(k, a)=\{w:|\arg (w+a)|<\pi-\arcsin k\} \cap\{|w|>1\}$.

Using this and a well known Jenkins result on circular symmetrization [6] together with the monotonicity property of the module, we obtain the inequalities

$$
\operatorname{Mod} D_{f} \leq \operatorname{Mod} D_{f}^{*} \leq \operatorname{Mod} D(k, a)
$$

where equality $\operatorname{Mod} D_{f}=\operatorname{Mod} D(k, a)$ holds if and only if $D_{f}$ is obtained from $D(k, a)$ by a rotation around the origin. From the relation

$$
\begin{gathered}
\operatorname{Mod} D_{f}=\operatorname{Mod} D\left(k, d_{0}\right)(=\log R), \\
\operatorname{Mod} D_{f} \leq \operatorname{Mod} D(k, a)
\end{gathered}
$$

and monotonicity of the module, we have

$$
a \geq d_{0},
$$

which implies the desired inequality (2.1). It is trivial that equality holds in (2.1) if and only if $D_{f}$ is $D\left(k, d_{0}\right)$ up to a rotation around the origin (see [6]).

Now we consider the case when $\Gamma$ does not contain the point at infinity. Without loss of generality we can assume $a=d(\Gamma, 0) \in \Gamma$. For a negative point $-d$ on $\Gamma$, the Möbius transformation $\zeta(w)=(1+d w) /(w+d)$ maps the points $a$ and $-d$ to $(1+a d) /(a+d)(<a)$ and the point at infinity, respectively. This means that the minimum of $d(\Gamma, 0)$ is attained (if and) only if $\Gamma$ contains the point at infinity. Therefore the inequality (2.1) holds even when $\Gamma$ does not contain the point at infinity.

3. Proof of the theorem. Let $w_{1}=r_{1} e^{i \theta}$ and $w_{2}=r_{2} e^{i(\pi-\theta)}$ $\left(=-r_{2} e^{-i \theta}\right)$ be the points on $\Gamma$ such that the segments $\left(e^{i \theta}, r_{1} e^{i \theta}\right)$ and $\left(-e^{-i \theta},-r_{2} e^{-i \theta}\right)$ are in $D_{f}$. Without loss of generality we can assume $r_{1}=a, r_{2}=a t(a>0, t \geq 1)$, because the case with $r_{1} \geq r_{2}$ can be proved analogously.

We consider the Möbius transformation

$$
h(w)=\frac{w_{1}}{\bar{w}_{1}} \cdot \frac{\bar{w}_{1} w-1}{w_{1}-w},
$$


which maps $f(A(R))$ onto $D\left(\Gamma^{\prime}\right)$ with inner boundary $\{|h|=1\}$ and outer boundary $\Gamma^{\prime}$. Since the chordal cross ratio is invariant under Möbius transformations, $\Gamma^{\prime}$ is also a $k$-circle. Substituting $w=w_{1}$ and $w=w_{2}$ into (3.1) we have the inequalities

$$
h\left(w_{1}\right)=\infty, \quad h\left(w_{2}\right)=-e^{2 i \theta} \frac{a^{2} t e^{-2 i \theta}+1}{a e^{i \theta}+a t e^{-i \theta}} .
$$

Now the fundamental lemma and $\left|h\left(w_{2}\right)\right| \geq d_{0}$ imply

$$
\begin{gathered}
\frac{1+2 a^{2} t \cos 2 \theta+a^{4} t^{2}}{a^{2}\left(1+2 t \cos 2 \theta+t^{2}\right)} \geq d_{0}^{2}, \\
a^{4} t^{2}-a^{2}\left(d_{0}^{2}\left(1+2 t \cos 2 \theta+t^{2}\right)-2 t \cos 2 \theta\right)+1 \geq 0 .
\end{gathered}
$$

From (3.4) we easily obtain either

$$
\begin{aligned}
a^{2} \geq & \frac{d_{0}^{2}\left(1+2 t \cos 2 \theta+t^{2}\right)-2 t \cos 2 \theta}{2 t^{2}} \\
& +\frac{\sqrt{\left(d_{0}^{2}\left(1+2 t \cos 2 \theta+t^{2}\right)-2 t \cos 2 \theta\right)^{2}-4 t^{2}}}{2 t^{2}}
\end{aligned}
$$

or

$$
\begin{aligned}
a^{2} \leq & \frac{d_{0}^{2}\left(1+2 t \cos 2 \theta+t^{2}\right)-2 t \cos 2 \theta}{2 t^{2}} \\
& -\frac{\sqrt{\left(d_{0}^{2}\left(1+2 t \cos 2 \theta+t^{2}\right)-2 t \cos 2 \theta\right)^{2}-4 t^{2}}}{2 t^{2}} .
\end{aligned}
$$

Using the fundamental lemma we now show that (3.6) never holds: Let $A$ and $B$ be positive constants such that $A \pm \sqrt{A^{2}-1}=\left(B \pm \sqrt{B^{2}-1}\right)^{2}$. Then $B=\sqrt{(A+1) / 2}$. If $A=\left(d_{0}^{2}\left(1+2 t \cos 2 \theta+t^{2}\right)-2 t \cos 2 \theta\right) / 2 t^{2}$, we have

$$
\begin{aligned}
B^{2} & =\frac{A+1}{2}=\frac{d_{0}^{2}\left(1+2 t \cos 2 \theta+t^{2}\right)-2 t \cos 2 \theta}{4 t^{2}}+\frac{1}{2} \\
& =d_{0}^{2} \frac{1+t^{2}}{4 t^{2}}+\frac{\left(d_{0}^{2}-1\right) \cos 2 \theta}{2 t}+\frac{1}{2} \leq \frac{d_{0}^{2}}{2}+\frac{d_{0}^{2}-1}{2}+\frac{1}{2}=d_{0}^{2} .
\end{aligned}
$$

On the other hand, the inequality (3.6) implies

$$
a^{2} \leq A-\sqrt{A^{2}-1}=\left(B-\sqrt{B^{2}-1}\right)^{2} \leq B^{2} \leq d_{0}^{2},
$$

contradicting $a \geq d_{0}>1$, because $a=d_{0}$ would imply $d_{0}=B=1$.

Now we utilize (3.5) to obtain

$$
\begin{aligned}
& \left(r_{1}+r_{2}\right)^{2}=a^{2}(1+t)^{2} \\
& \quad \geq \frac{(1+t)^{2}}{2 t^{2}}\left(d_{0}^{2}\left(1+2 t \cos 2 \theta+t^{2}\right)-2 t \cos 2 \theta\right. \\
& \left.\quad+\sqrt{\left(d_{0}^{2}\left(1+2 t \cos 2 \theta+t^{2}\right)-2 t \cos 2 \theta\right)^{2}-4 t^{2}}\right)
\end{aligned}
$$




$$
\begin{aligned}
= & \frac{(1+t)^{2}}{t}\left(d_{0}^{2}\left(\frac{1+t^{2}}{2 t}+\cos 2 \theta\right)-\cos 2 \theta\right. \\
& \left.+\sqrt{\left(d_{0}^{2}\left(\frac{1+t^{2}}{2 t}+\cos 2 \theta\right)-\cos 2 \theta\right)^{2}-1}\right) \\
\geq & 4\left(d_{0}^{2}(1+\cos 2 \theta)-\cos 2 \theta+\sqrt{\left(d_{0}^{2}(1+\cos 2 \theta)-\cos 2 \theta\right)^{2}-1}\right) \\
= & 4\left(d_{1}+\sqrt{d_{1}^{2}-1}\right)^{2} \quad\left(d_{1}=\sqrt{d_{0}^{2} \cos ^{2} \theta+\sin ^{2} \theta}\right),
\end{aligned}
$$

which implies $r_{1}+r_{2} \geq 2\left(d_{1}+\sqrt{d_{1}^{2}-1}\right)$. Since $\ell(\theta) \geq r_{1}$ and $\ell(\pi-\theta) \geq r_{2}$, we obtain the desired inequality (1.3). Using the fundamental lemma and (3.9), we conclude that equality in (1.3) is attained only if $t=1, r_{1}=r_{2}=$ $\ell(\theta)=\ell(\pi-\theta)=d_{1}+\sqrt{d_{1}^{2}-1}$, and only if $f(A(R))$ is a rotation of $D\left(k, d_{0}\right)$ around the origin.

It follows trivially from the fundamental lemma that

$$
\ell(\theta) \geq d_{0}, \quad \ell(\pi-\theta) \geq d_{0} .
$$

For $\arccos \left(\sqrt{d_{0}^{2}-1} /\left(2 d_{0}\right)\right)<\theta \leq \pi / 2$, by a simple calculation, we conclude that

$$
d_{1}+\sqrt{d_{1}^{2}-1}<d_{0},
$$

which implies that the inequality (1.4) is better than (1.3) in this case.

Next we discuss the case of equality in (1.3). For the case of $w_{1}=a_{0} e^{i \theta}$, $w_{2}=-a_{0} e^{-i \theta}\left(a_{0}=d_{1}+\sqrt{d_{1}^{2}-1}\right)$, we have

$$
\begin{gathered}
h\left(w_{2}\right)=-e^{2 i \theta} \frac{1+a_{0}^{2} e^{-2 i \theta}}{a_{0}\left(e^{i \theta}+e^{-i \theta}\right)}=-\frac{a_{0}^{2}+e^{2 i \theta}}{2 a_{0} \cos \theta}=-d_{0} e^{i \beta} \quad(\beta \text { real }), \\
a_{0}^{2}+e^{2 i \theta}=2 d_{0} a_{0} e^{i \beta} \cos \theta \\
\sin 2 \theta=2 d_{0} a_{0} \sin \beta \cos \theta \\
\sin \theta=d_{0} a_{0} \sin \beta \\
\beta=\arcsin \left(\sin \theta /\left(d_{0} a_{0}\right)\right) \quad(0 \leq \beta<\theta) .
\end{gathered}
$$

Now we determine the value $\theta_{0}$ mentioned in the theorem, as follows: For the extremal function $F(z)$, the point $h(\infty)=-w_{1}=-a_{0} e^{i \theta}$ must be contained in the complement of $e^{i \beta} D\left(k, d_{0}\right)$, because the extremal function must be conformal. Considering the rotation around the origin through $\pi-\beta$, we see that the point $a_{0} e^{i(\theta-\beta)}$ must lie in the closed domain $\{w$ : $\left.\left|\arg \left(w-d_{0}\right)\right| \leq \arcsin k\right\}$. We consider two functions of the angle $\theta$,

$$
\begin{gathered}
Y_{1}(\theta)=a_{0}=\sqrt{\left(d_{0}^{2}-1\right) \cos ^{2} \theta+1}+\sqrt{\left(d_{0}^{2}-1\right) \cos ^{2} \theta}, \\
Y_{2}(\theta)=d_{0} k / \sin \left(\theta_{2}-\theta\right) \quad\left(\theta_{2}=\arcsin k\right),
\end{gathered}
$$

where (3.14) represents the rays $\left\{w:\left|\arg \left(w-d_{0}\right)\right|=\arcsin k\right\}$ in polar coordinates $\left(Y_{2}, \theta\right)$. The functions $Y=Y_{1}(\theta)$ and $Y=Y_{2}(\theta)$ are, respectively, 
strictly decreasing and increasing, and their values run from $d_{0}+\sqrt{d_{0}^{2}-1}$ to $1(0 \leq \theta \leq \pi / 2)$ and from $d_{0}$ to $\infty\left(0 \leq \theta \leq \theta_{2}\right)$, respectively. Therefore the curves $Y=Y_{1}(\theta)$ and $Y=Y_{2}(\theta)$ intersect at some point $\theta=\theta_{3}\left(<\theta_{2}\right)$. Since

$$
a_{0}=\sqrt{\left(1-d_{0}^{2}\right) \sin ^{2} \theta+d_{0}^{2}}+\sqrt{\left(1-d_{0}^{2}\right) \sin ^{2} \theta+d_{0}^{2}-1}
$$

(which implies that $\beta=\beta(\theta)$ is a strictly decreasing function of $\theta$ for $0 \leq$ $\theta \leq \pi / 2)$ and $\beta(\theta)<\theta$, the function $\theta-\beta(\theta)$ is non-negative and strictly increasing for $0 \leq \theta \leq \pi / 2$ and varies from 0 to $\pi / 2-\arcsin \left(1 / d_{0}\right)$ there. Therefore there exists a constant $\theta_{0}$ such that $0 \leq \theta-\beta \leq \theta_{3}$ for $0 \leq \theta \leq \theta_{0}$. Then the point $a_{0} e^{i(\theta-\beta)}$ is contained in $\left\{w:\left|\arg \left(w-\bar{d}_{0}\right)\right| \leq \arcsin k\right\}$ for $0 \leq \theta \leq \theta_{0}$.

Since $T(w)$ is the inverse function of (3.1) the function $F(z)$ maps $A(R)$ onto the extremal domain which has two points $w_{1}=a_{0} e^{i \theta}$ and $w_{2}=$ $a_{0} e^{i(\pi-\theta)}$ on the boundary $F(|z|=R)$ for $0 \leq \theta \leq \theta_{0}$, and so the theorem has been verified.

\section{References}

[1] L. V. Ahlfors, Quasiconformal reflections, Acta Math. 109 (1963), 291-301.

[2] D. K. Blevins, Conformal mappings of domains bounded by quasiconformal circles, Duke Math. J. 40 (1973), 877-883.

[3] - Harmonic measure and domains bounded by quasiconformal circles, Proc. Amer. Math. Soc. 41 (1973), 559-564.

[4] - Covering theorems for univalent functions mapping onto domains bounded by quasiconformal circles, Canad. J. Math. 28 (1976), 627-631.

[5] W. K. Hayman, Multivalent Functions, Cambridge Univ. Press, 1958.

[6] J. A. Jenkins, Some uniqueness results in the theory of symmetrization, Ann. of Math. 61 (1955), 106-115.

[7] O. Lehto and K. I. Virtanen, Quasiconformal Mappings in the Plane, second ed., Springer, 1973.

[8] I. P. Mityuk, Principle of symmetrization for the annulus and some of its applications, Sibirsk. Mat. Zh. 6 (1965), 1282-1291 (in Russian).

DEPARTMENT OF APPLIED MATHEMATICS

KOBE UNIVERSITY OF MERCANTILE MARINE

FUKAE MINAMIMACHI 5-1-1

HIGASHINADA-KU

KOBE, JAPAN 658 